\title{
Gradhiva
}

GRADHIV

Revue d'anthropologie et d'histoire des arts

17 | 2013

L'esthétique du geste technique

\section{À main levée. La scarification comme œuvre}

Freehand - An African example of bodily scarification

\section{Michèle Coquet}

\section{(2) OpenEdition}

Journals

Édition électronique

URL : http://journals.openedition.org/gradhiva/2627

DOI : $10.4000 /$ gradhiva.2627

ISSN : 1760-849X

\section{Éditeur}

Musée du quai Branly Jacques Chirac

\section{Édition imprimée}

Date de publication : 16 mai 2013

Pagination : 94-117

ISBN : 978-2-35744-049-74

ISSN : 0764-8928

Référence électronique

Michèle Coquet, «À main levée. La scarification comme œuvre », Gradhiva [En ligne], 17 | 2013, mis en ligne le 28 mai 2013, consulté le 19 avril 2019. URL : http://journals.openedition.org/gradhiva/2627 ; DOI : 10.4000/gradhiva.2627 


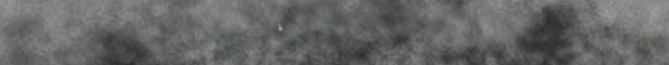

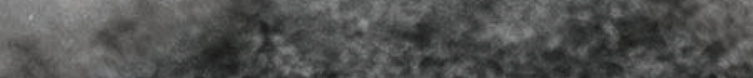

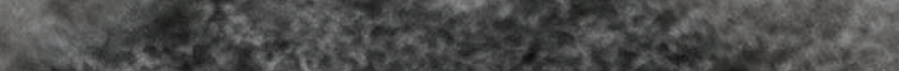

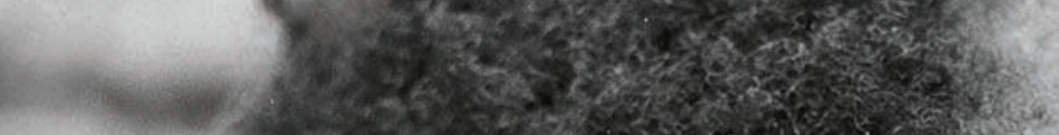

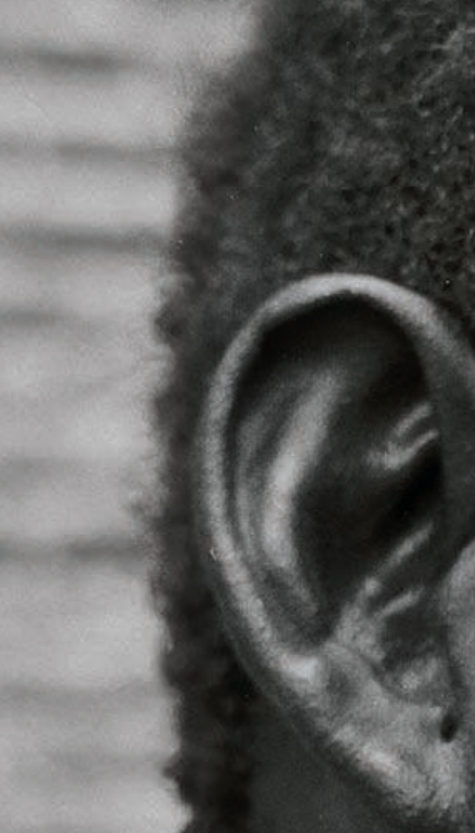

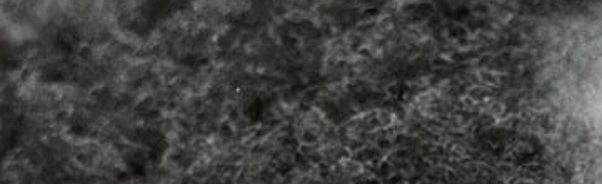

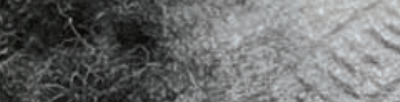

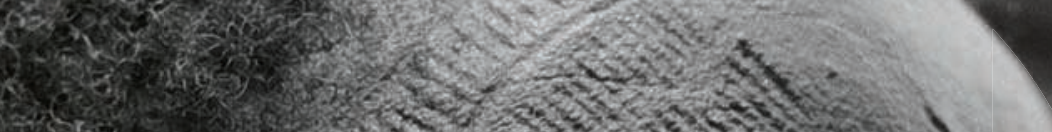

$\frac{1}{6}$
0
0
0
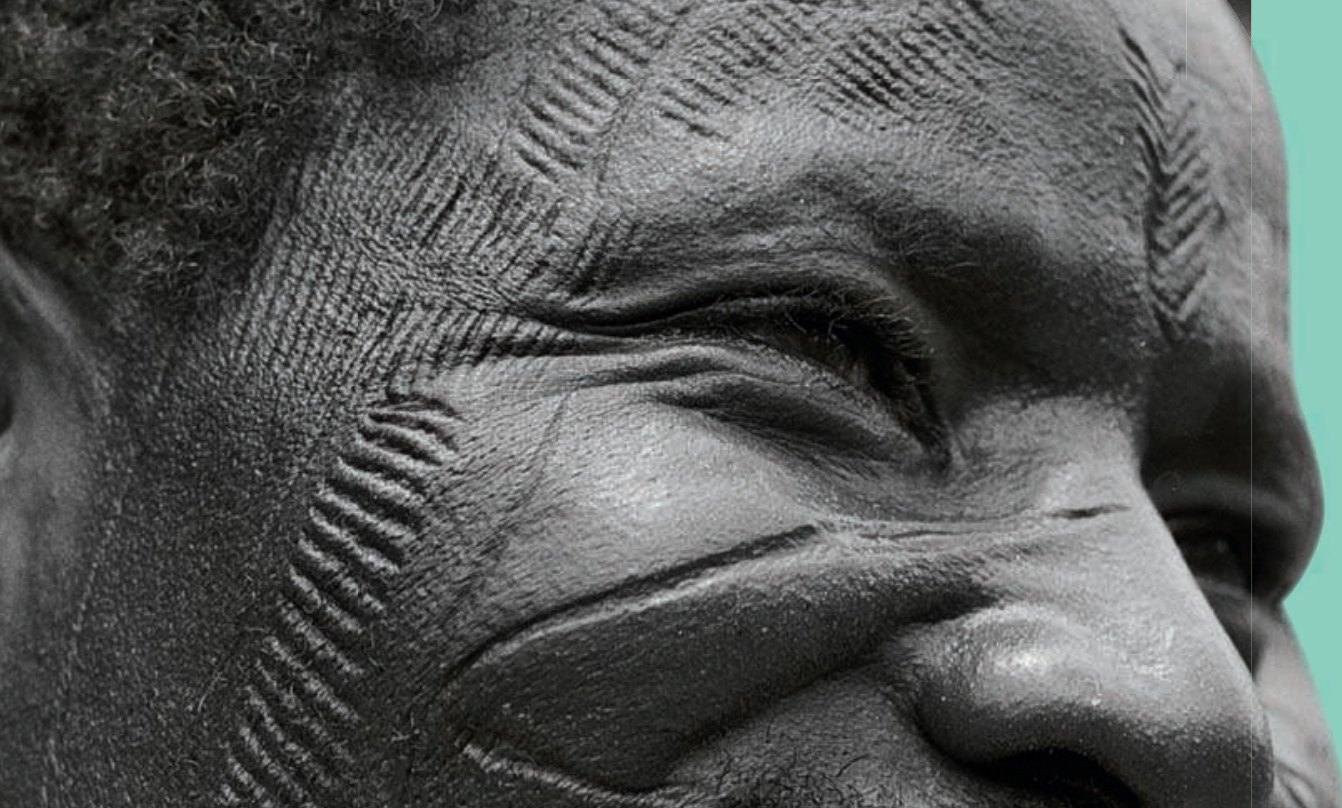

$x^{3}, x^{2}$

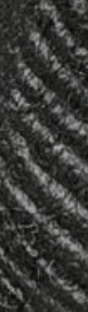
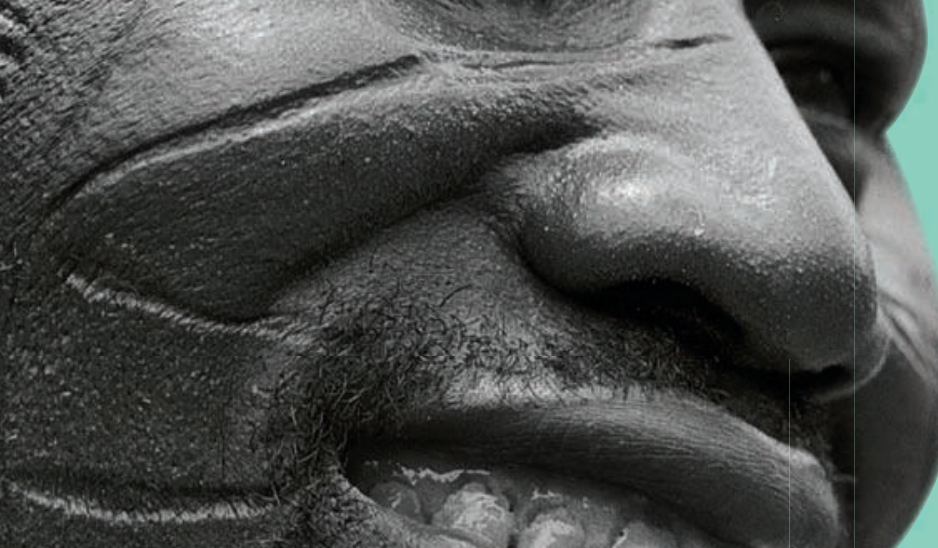

\%

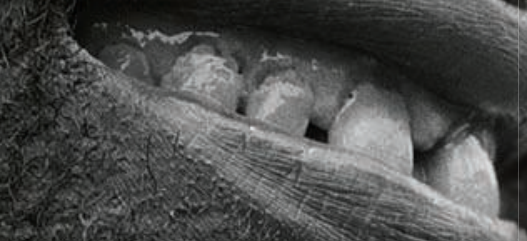

(3) 


\section{À main levée}

La scarification comme cuvre

par Michèle Coquet

La maîtrise d'un geste technique, et de ce qu'il produit, prédispose à l'admiration et à la valorisation esthétique tout observateur ignorant des règles présidant à sa réalisation. L'ignorance ne fait même qu'en accroître l'intensité. Les femmes bwaba (Burkina Faso) entretenaient soigneusement le mystère entourant leur savoir-faire relatif à l'inscription sur la peau de complexes compositions graphiques. Savoir graver une image dans la matière vivante des corps, savoir la composer puis la soigner de manière à garantir le résultat, avoir le courage d'infliger des blessures douloureuses et d'être confrontées aux risques d'infection constituaient quelques-unes de leurs compétences, à la fois techniques et morales. Ces femmes jouissaient toutes d'une grande estime et certaines d'entre elles d'une renommée qui s'étendait bien au-delà de leur village. C'est seulement lorsque ces compétences avaient été démontrées que leur renommée s'installait, entraînant dans son sillage la reconnaissance de la singularité de leur œuvre. Certaines scarificatrices étaient alors considérées comme des auteurs possédant leur propre style, susceptible d'être décrit en tant que tel. 
1. Reprenant sans le citer la notion forgée par l'historien de l'art suisse Heinrich Wölfflin (1864-1945).
La maîtrise du geste et sa matérialisation dans l'œuvre achevée prédisposent à l'admiration tout observateur ignorant des règles présidant à sa réalisation. II y reconnaît la marque du métier, à savoir l'habileté et l'intelligence techniques que confère sa pratique lorsqu'elle s'inscrit dans la durée, mais n'en possède pas les secrets, ce qui accroît en proportion son émerveillement. À titre d'exemple, je rappellerai que, chez nombre de nos contemporains, cette identification est en grande partie à l'origine de l'engouement pour les œuvres d'art classique où la marque du métier est immédiatement intelligible. Je pense ici aux peintres de nature morte de la Hollande du $\mathrm{xvII}^{\mathrm{e}}$ siècle qui atteignirent un degré de virtuosité tel qu'ils ont rendues tangibles la texture ou la consistance de matériaux qui semblaient défier toute possibilité de reproduction par l'image comme la transparence du verre, l'éclat nacré du coquillage, le chatoiement du brocart, le brouillard de dentelle des cols. Aussi le spectateur pouvait-il juger sur pièce, l'évidence du fait présupposant les savoir-faire. À l'inverse, lorsque l'identification du métier devient impossible, comme c'est souvent le cas dans les œuvres d'art contemporain où l'artiste choisit de ne plus rendre perceptible une quelconque compétence technique, voire de la dénier intentionnellement, l'incompréhension et le rejet s'installent.

II en va de la peinture hollandaise comme des autres artefacts: la capacité, présupposant une connaissance même très partielle de certains actes techniques, à apprécier la qualité de leur facture ou de leur dessin est une des conditions de leur valorisation esthétique. Franz Boas ne disait pas autre chose lorsqu'il affirmait que le "sentiment de la forme", chez l'artisan comme chez son client, "est inextricablement lié à l'expérience technique", précisant encore que «l'intérêt pour les formes est directement lié aux impressions que celles-ci suscitent en nous" (Boas 2003 [1927]: 41 et 93). L'action qu'exercent sur celui qui les regarde une peinture, un décor, les formes d'un objet quelconque, et que Boas nomme le "sentiment de la forme ${ }^{1}$ ", est cependant en grande partie déterminée par le contexte dans lequel elle s'exerce. Dans le cas de la peinture hollandaise, destinée à orner les intérieurs de la bourgeoisie des riches cités marchandes d'Amsterdam ou de Haarlem, intervenaient d'autres paramètres essentiels, telles la fluctuation de la mode et ce qui s'ensuivait, la cote du peintre.

Je m'intéresserai ici aux critères d'évaluation d'un geste technique singulier, le marquage des corps par scarification tel qu'il se pratiquait dans toute l'Afrique subsaharienne, et aux conditions de sa réalisation. Compte tenu de la spécificité des œuvres graphiques ainsi obtenues - prenant forme non en surface d'un objet inanimé mais dans un corps de chair vivant et souffrant -, d'autres éléments que l'excellence technique et les mystères entourant leur exécution façonnent la perception qu'en ont les intéressés. Celle-ci passe par l'expérience de la douleur physique et de la souffrance morale. II faut que ces épreuves aient été dignement surmontées pour qu'advienne la reconnaissance des qualités esthétiques du geste du graveur de peau et de son œuvre. Les marques de la douleur endurée, visible et quantifiable par le nombre des scarifications, provoquent l'admiration lorsqu'elles sont abondantes, révélant la teneur du courage et de la volonté de celui qui les reçoit. Le sentiment de la beauté naît alors de la conscience que ces deux dispositions sont réunies, tant chez celui qui agit que chez celui qui subit. 
Lacompréhension d'une pratique graphique decetype, profondément étrangère à la tradition occidentale de la figuration, nécessite que soit auparavant décrit le cadre sociologique de son accomplissement.

Les femmes de forgerons des Bwaba, une société d'agriculteurs du Burkina Faso, entretenaient soigneusement elles aussi le mystère autour de leur savoir-faire de scarificatrices, impliquant de complexes compositions graphiques. Ce faisant, elles procédaient comme le voulait la coutume chez ces artisans, à la fois prestataires de services indispensables à la survie de la collectivité villageoise et détenteurs de savoirs technologiques tenus aussi secrets que ceux de nos miroitiers, émailleurs, teinturiers et autres maîtres artisans médiévaux. Savoir graver une image dans la matière vivante des corps, la composer puis la soigner de manière à garantir l'excellence du résultat, avoir le courage d'infliger des blessures douloureuses et être confrontées aux risques d'infection constituaient quelques-unes de leurs compétences, tout autant techniques que morales, définissant la nature particulière de leur métier. Ces femmes jouissaient toutes d'une grande estime et la renommée de certaines s'étendait bien au-delà de leur village, entraînant dans son sillage la reconnaissance de la singularité de leur œuvre; elles étaient alors considérées comme des auteurs possédant un style propre, susceptible d'être décrit en tant que tel.

Les Bwaba composent une société segmentaire associant, au premier chef, deux groupes: les «gens de la terre» - les cultivateurs, les buaba proprement dits - et des migrants regardés comme des étrangers, les «gens du fer» - les forgerons, ou likiba. Les ethnographies relatives à cette aire, corroborant la sociologie autochtone, ont décrit la dualité de cette structure: la viabilité de cette société agricole repose sur une coopération active des cultivateurs et des métallurgistes. En échange de la collaboration technologique, thérapeutique, magique et cultuelle de ces derniers, les cultivateurs leur ont donné une portion de sol où planter leur atelier, mobile par définition, et où construire leurs habitations: autrefois, ils leur cédaient également une part de leurs récoltes.

Les gens de la terre se présentent comme les descendants, dans la glose locale, des premiers occupants du pays. Du lignage fondateur d'un village est issu le «maître de la terre», chargé de l'autel dédié à cette puissance. Lui revient, plus largement, la gestion foncière, économique et rituelle du territoire villageois, de ses arbres, de ses terres cultivées, du sous-sol où sont ensevelis les morts, des eaux qui y circulent; sa gestion concerne autant la culture que la chasse, la pêche ou la cueillette de certains fruits. Les gens de la terre sont aussi maîtres de l'air et des eaux, garants de l'abondance des pluies, de la fertilité du sol et des récoltes. La terre, instance de contrôle social par excellence, est entendue à la fois comme le matériau et l'étendue travaillés par les humains qui y font croître les végétaux nourriciers, et comme une puissance formée d'un ensemble de forces redoutables dont dépend leur survie.

Au sein de cette société, les forgerons forment des lignages d'artisans statutaires, endogames, comme on en rencontre dans toute l'Afrique de l'Ouest: ils cumulent un ensemble de compétences qui, ailleurs, sont souvent 


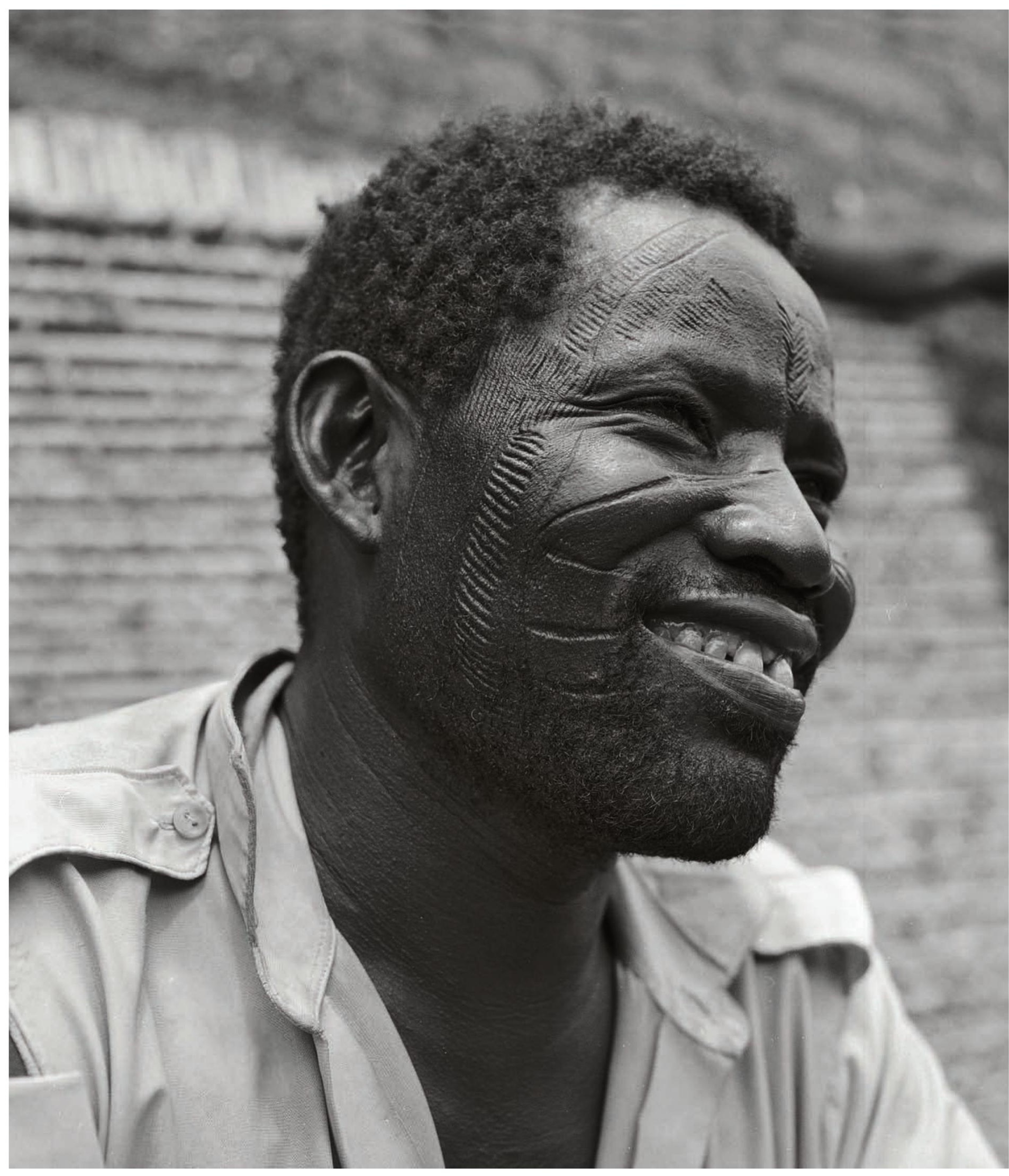




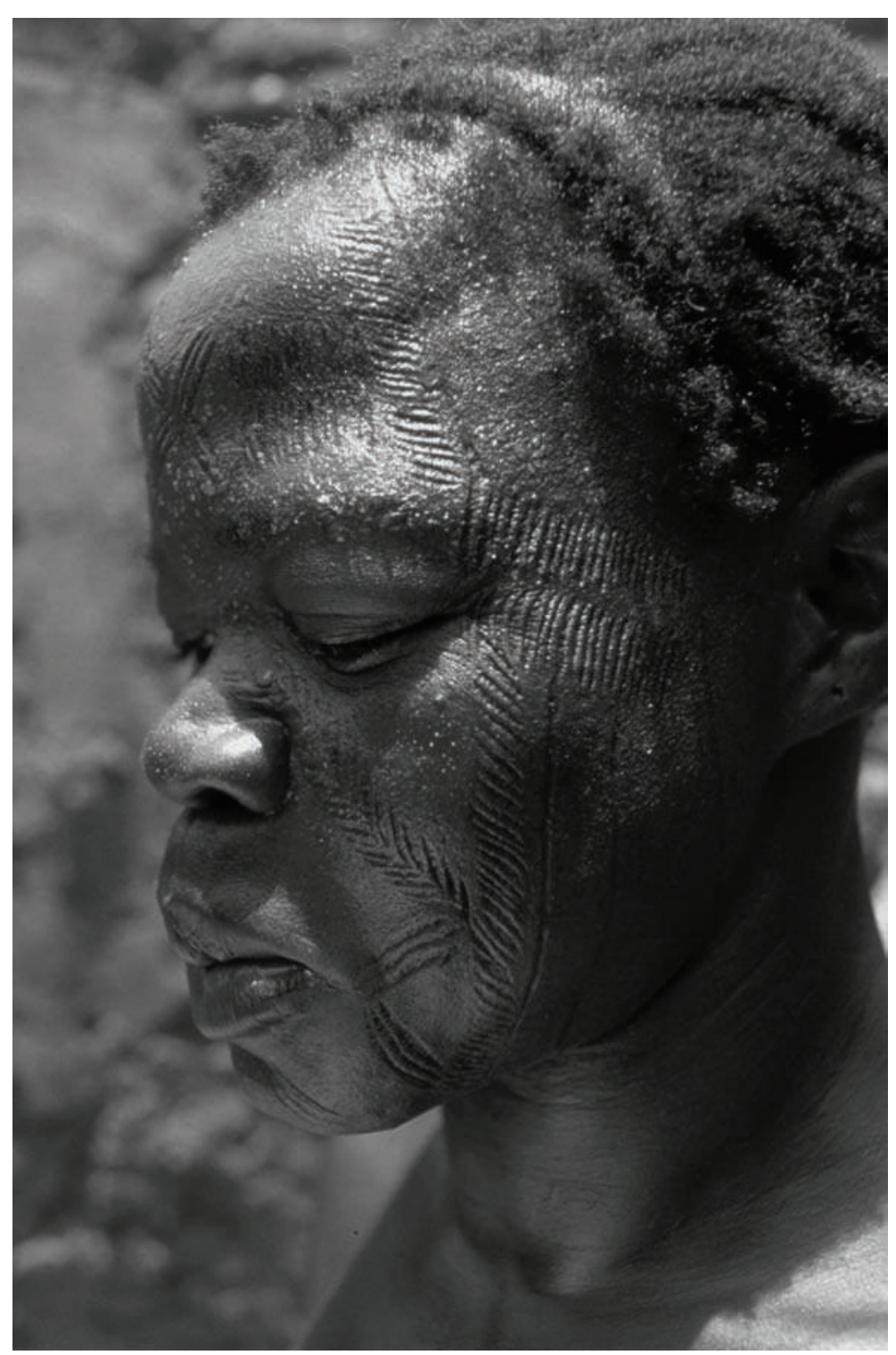

ci-dessus

fig. 2

Femme du village

de Boni, Burkina Faso, 1983.

(c) Photo Michèle Coquet.

\section{ci-contre}

fig. 1

Homme du village

de Boni, Burkina Faso, 1981.

(c) Photo Michèle Coquet. 
2. L'imparfait est ici de rigueur, ces pratiques ayant à ce jour quasi disparu. En Afrique occidentale francophone, elles ont été très rapidement interdites après les indépendances. Les fortes peines auxquelles ont été condamnés les auteurs des incisions et les bouleversements sociologiques que connurent ces sociétés rurales ont dissuadé progressivement les villageois de soumettre leurs enfants à ces épreuves. Je m'appuie ici sur les données ethnographiques que j'ai recueillies durant les années 1980 dans la région de Houndé.

L'opération de la scarification ayant été proscrite, elle n'était alors plus perpétrée que dans la clandestinité. En conséquence, je n'ai pas pu l'observer directement. le fait de corporations d'artisans distinctes. Compétences techniques d'une part: ils fondaient autrefois le minerai de fer, fabriquaient les armes et la poudre pour les fusils. Aujourd'hui encore, ils forgent et réparent l'outillage agricole et toute la coutellerie, travaillent les alliages de cuivre, modèlent la cire et l'argile, cisèlent et gravent. Ils sculptaient auparavant tous les objets mobiliers (dont les masques) et les ustensiles domestiques; ils tissent toujours d'amples couvertures à damiers ou des pagnes. Leurs femmes façonnent les poteries.

Compétences rituelles et cultuelles d'autre part: alors que leurs époux, fossoyeurs, procèdent à la séparation définitive du mort d'avec sa communauté, les femmes ont pour tâche, en tant qu'accoucheuses, d'accueillir chaque nouveau-né. Elles excisent les filles et autrefois scarifiaient les corps. Aux hommes, desservants du culte agraire communautaire, le culte de doo, reviennent également la maîtrise d'éléments atmosphériques comme la foudre, une connaissance approfondie des propriétés thérapeutiques des plantes et un pouvoir de médiation avec certaines puissances de la brousse ou du sous-sol associé à leurs travaux d'excavation (puits et mines).

La spécificité des connaissances et des savoir-faire des artisans était préservée par l'obligation rigoureuse de ne pas divulguer leurs secrets, qui concernaient tant les opérations de fabrication que leur exégèse. Le mystère qui entourait leurs pratiques et leurs personnes, leur allochtonie, les contraintes de l'endogamie, le tout construisait l'altérité des forgerons que les comportements à leur égard contribuaient à rendre effective. C'est une femme, disent encore aujourd'hui les cultivateurs du forgeron, car il ne peut faire la guerre. Maître du fer et du feu, il ne peut ni blesser ni être blessé par le fer lors des conflits. Désigné comme «celui qui pardonne» (iankaro), il est en son pouvoir de mettre hors jeu ceux qui menacent la stabilité de l'ordre social. Si le qualificatif de «femme» désigne la fonction de pacificateur que la communauté alloue aux forgerons, il en affirme simultanément et avant tout l'irréductible différence et la position hégémonique de ceux qui l'utilisent. Et c'est aux femmes des forgerons que les cultivateurs avaient confié la tâche, non seulement d'accoucher leurs épouses, mais encore d'inciser tous les visages et les torses des membres de la communauté. Elles seules, fortes de la compétence morale que leur conférait leur statut, avaient été considérées à même de pouvoir, dans un même mouvement, à la fois infliger et apaiser la douleur extrême que provoquait la blessure de leur lame chez les enfants et les adolescents de toute la communauté.

L'ornementation par scarification de l'épiderme a constitué pour les Bwaba un mode d'expression graphique très sophistiqué dont on ne trouvait pas d'équivalent dans le décor de leurs objets ${ }^{2}$. Celui des poteries ou des réalisations en laiton ou en bois, les masques en particulier, bien moins complexe, requérait peu d'habileté pour sa réalisation. Si la plupart des ethnies voisines pratiquaient les scarifications, aucune n'avait élaboré des représentations d'une finesse comparable. Entre tous les supports possibles que leur offrait le monde des objets fabriqués, les Bwaba avaient donc choisi, comme surface et matière d'inscription privilégiées, celles de leur corps vivant. À travers l'acte de marquage et les images qui en résultaient, étaient réactualisés puis visuellement exprimés par inscription 
dans l'épiderme les fondements économiques, politiques, moraux, religieux et esthétiques de leur société.

Dans les années 1980, tous les Bwaba âgés de plus de 20 ans, qu'ils soient cultivateurs ou artisans, présentaient encore un visage orné d'incisions (Coquet 1994). Mais seuls les jeunes gens, et en particulier les jeunes filles, nés dans des lignages de cultivateurs fondateurs de villages arboraient des torses scarifiés. L'incision des motifs du visage concernait l'ensemble des enfants en âge d'être opérés (vers 5 ans), amenés au petit matin chez le forgeron par le doyen de leur lignage. La journée était réservée à la scarification des enfants d'un seul et même lignage, une règle qui nécessitait l'étalement de l'opération sur plusieurs jours lorsque le village comprenait plusieurs lignages. La scarification du corps qui intervenait à la puberté ne se déroulait pas de la même manière puisque le jeune homme ou la jeune fille se rendait généralement seul chez le forgeron ${ }^{3}$. L'opération avait lieu durant les mois de décembre à février, lorsque règne encore à l'aube une relative fraîcheur favorable, dit-on, à la guérison des plaies. Cette période était aussi celle qui précédait l'ouverture des grandes festivités religieuses et marquait le commencement de la nouvelle année, juste après la dernière récolte de mil.

Les images de torse constituaient l'emblème visuel d'une appartenance lignagère immédiatement compréhensible comme tel par tous les villageois, qui n'ignoraient pas que seuls les gens de la terre exposaient ainsi la marque de leurs droits fonciers. Elles distinguaient donc ceux qui étaient nés dans un lignage de fondateurs en inscrivant dans leur chair le signe d'un lien contractuel établi en des temps anciens entre des puissances de la terre et des hommes (l'ancêtre et les siens), lien sans lequel l'existence d'aucune communauté humaine quelle qu'elle soit n'aurait été pensable. Autrement dit, chaque personne arborant sur le torse une image de ce type portait inscrite dans son épiderme la marque d'un contrat qui la liait ontologiquement à un territoire.

Ces images étaient relayées par certains motifs incisés sur le visage qui désignaient eux aussi l'appartenance du porteur au lignage fondateur. Pour les chefs d'un tel lignage, spécialement pour les maîtres de la terre, elles étaient plus qu'un élément signalétique. Ils savaient, sans pour autant posséder un code d'interprétation dont la connaissance revenait aux seuls forgerons, qu'elles transcrivaient précisément certaines données du récit étiologique légitimant leur statut et leurs droits (Coquet 2006).

Lorsque je découvris ces images sur le corps de mes hôtes, je fus saisie non seulement par leur profusion mais également par l'extrême régularité de leur tracé. J'eus le sentiment que, au visage donné par la nature, s'en superposait un autre, plus présent, dont la prégnance me pénétrait à mon tour, m'empêchant de voir leur «vrai » visage. Cette impression première s'estompa progressivement, sans pour autant jamais disparaître, à mesure que je me familiarisais avec les personnes et apprenais à apprécier dans certains cas la cohérence visuelle des traits, qu'ils soient naturels ou incisés. Quant aux scarifications du torse, je ne les perçus pas immédiatement comme des compositions structurées, encore moins comme des images
3. D'autres motifs pouvaient être ajoutés plus tard, en particulier sur les bras. Le marquage du visage comme du torse était rétribué en cauris, la monnaie traditionnelle dont l'usage a été conservé jusqu'à aujourd'hui pour le paiement d'actes rituels. 


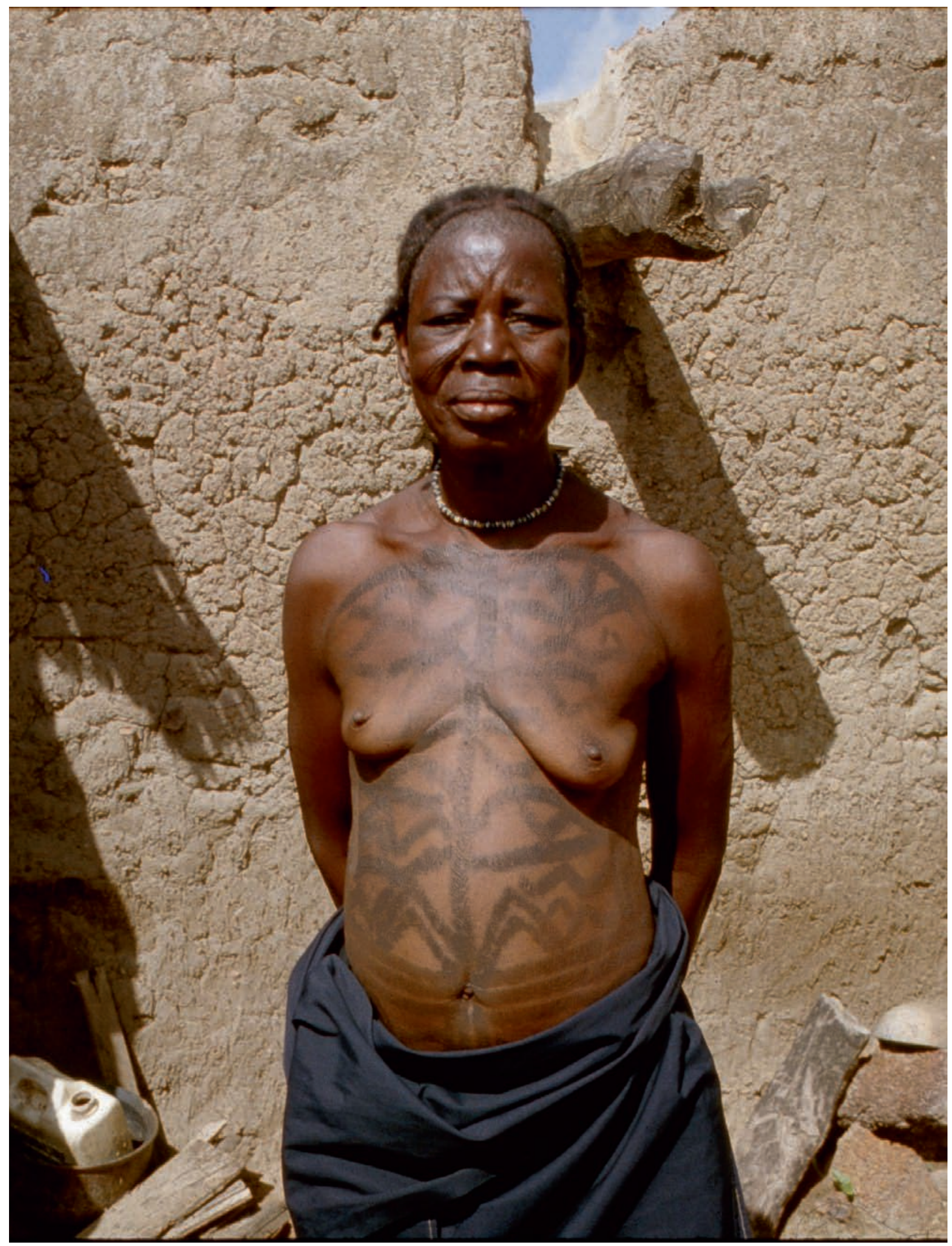




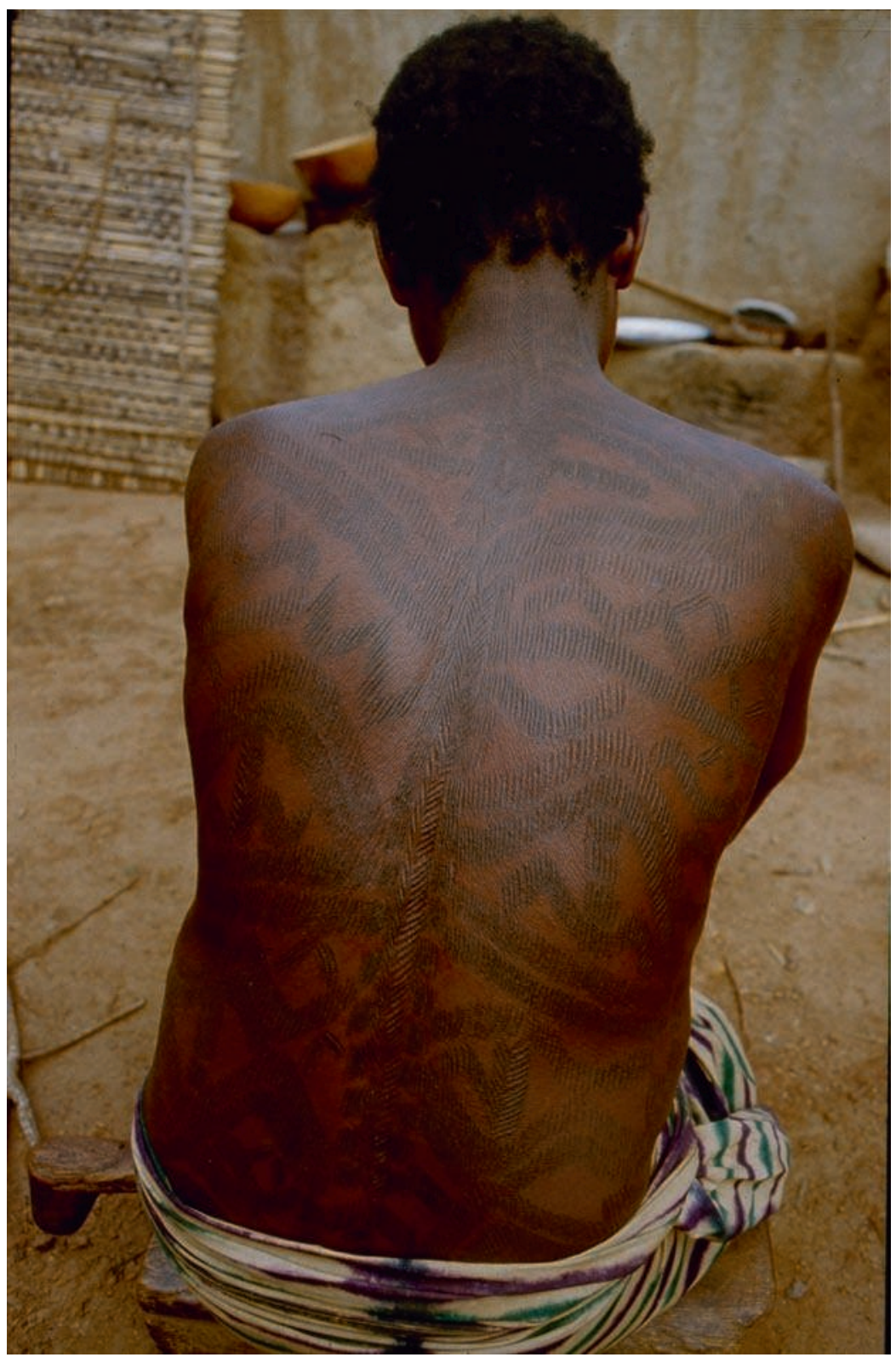

ci-contre

et ci-dessus

fig. 3

Femmes du village de

Mamboué, Burkina Faso, 1981.

(C) Photos Michèle Coquet. 


\section{ci-contre}

fig. 4

Séance de scarification d'une femme du village de

Yalka, Burkina Faso, 1971.

(c) Photos Suzanne Lallemand. car je n'y voyais aucune représentation, mais seulement un gracieux décor réticulé couvrant dans certains cas de son voile bleuté tout l'épiderme du buste. Je fus donc un certain temps dans l'incapacité de les décrypter (fig.1, 2 et 3 , planche I).

L'étude de ces compositions scarifiées exigeant que j'en fasse des relevés graphiques, j'en eus, en tentant de les reproduire, une meilleure intelligence. Ce qui m'était apparu de prime abord confus, incohérent et vaguement répétitif, acquit par ce biais clarté et précision. Les règles de composition se révélèrent à mon entendement, et là où je ne voyais que des décors semblables, je discernai bientôt des singularités et sus identifier des différences de style. À chacun était attribué un nom, celui de la femme dont il était la signature. Le style n'était pas tant identifiable par la composition générale des motifs obéissant à des règles canoniques qu'à la facture des incisions, leur profondeur, leur densité et leur régularité. Cet exercice préalable me permit également, toutes proportions gardées toutefois puisque je l'effectuai sur une feuille de papier, de prendre la mesure des difficultés techniques que comportait ce travail d'inscription dans la matière courbe, souple, élastique et vivante d'un épiderme.

\section{L'exercice du savoir-faire}

Les enfants de forgerons, filles et garçons, grandissent dans l'atelier, un lieu où les non-forgerons ne peuvent pénétrer sans y être invités. Ils y passent une partie de leurs journées; tandis que les plus jeunes folâtrent en se roulant dans la cendre des foyers, leurs aînés des deux sexes, dès l'âge de 6-7 ans, s'initient aux premiers actes techniques: maniement rythmé des peaux des poteries pulsant l'air dans les tuyères pour attiser le feu et préhension de la pince tenant la pièce de métal portée au rouge que le forgeron martèle pour la former constituent les deux apprentissages propres à cet âge. Dans l'atelier, la transmission régulée des savoir-faire est le produit d'une lente imprégnation fondée sur l'imitation, nécessitant deux qualités principales chez les jeunes apprentis: une immense patience, puisque l'acquisition des compétences est très progressive et ne s'accompagne d'aucun commentaire, et le sens de l'observation. Seuls les garçons peuvent être formés au travail de la forge proprement dit.

C'est donc à l'extérieur de l'atelier que se transmettait le savoirfaire concernant la scarification, sur la scène même de l'opération, et il ne concernait que les femmes. II revenait en principe à la femme du doyen du lignage des forgerons de remplir la fonction de scarificatrice et d'instruire la jeune femme destinée à lui succéder, épouse, idéalement, de son premier fils. Ce schéma de transmission était évidemment loin d'être respecté, car c'était moins la position matrimoniale occupée par telle ou telle jeune femme mariée dans le lignage qui importait que certaines aptitudes à satisfaire cette fonction particulière, évaluées par son instructrice en dehors du cadre de l'opération elle-même, dans les actes de la vie quotidienne: constance de I'humeur, patience et application, exactitude, sang-froid et, dans une moindre mesure, habileté manuelle évaluée à l'aune d'autres pratiques comme celle de la poterie. 


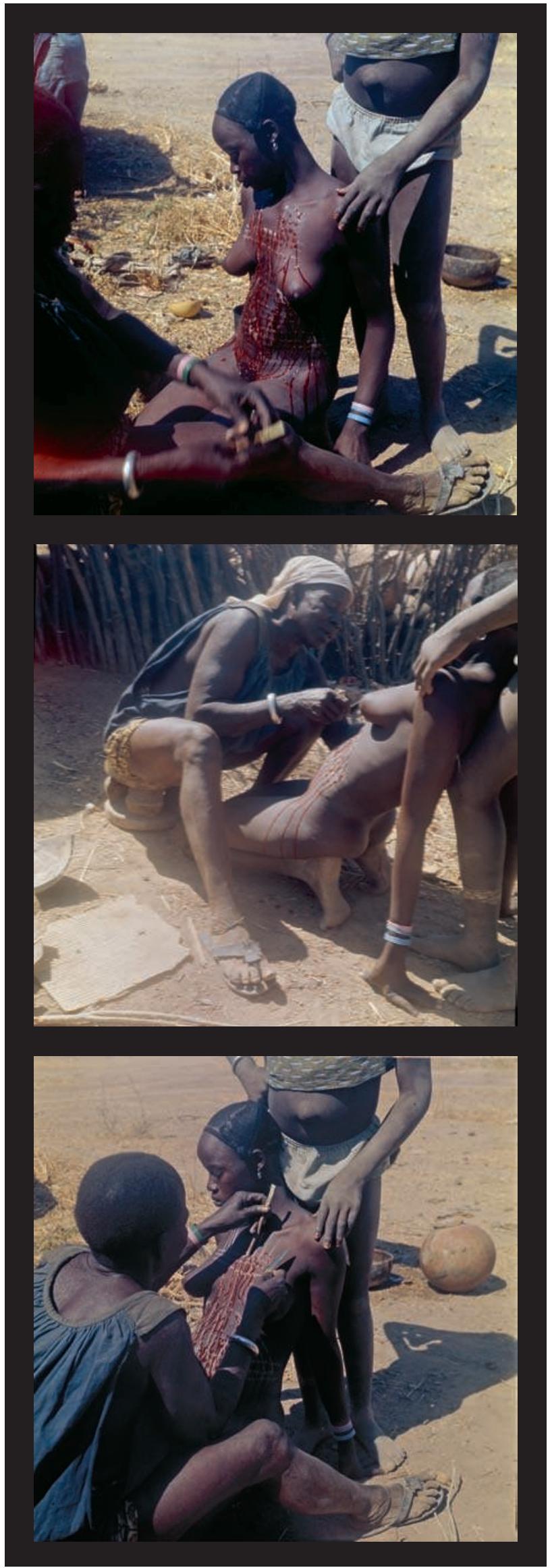


4. Ces différentes opérations se déroulaient en dehors de l'initiation.
La jeune femme élue pour ces différentes qualités était ensuite destinée à assister la scarificatrice. Son rôle consistait alors essentiellement à tenir la tête des enfants lorsqu'on leur scarifiait le visage ou à soutenir les jeunes gens par sa présence attentive durant le marquage de leur torse. Elle ne pouvait en aucun cas effectuer elle-même les incisions ni manipuler les lames avant d'avoir été intronisée à cette charge au décès de sa formatrice. L'observation, des années durant, des modalités d'exécution des scarifications dans l'épiderme constituait l'essentiel de son éducation. Par ce biais, elle acquérait la conscience des principes de composition des images, en particulier pour celles, parfois très profuses, qui étaient inscrites sur le torse. Elle apprenait comment tenir la lame et couper l'épiderme, jaugeait la pression de la main sur la peau; et lorsqu'elle maintenait sur les cuisses de l'opératrice la tête de l'enfant présentée de profil (une moitié du visage était scarifiée, puis l'autre), elle mémorisait à la fois l'ordre dans lequel étaient effectués les motifs et les différents éléments du corpus graphique, apprenant progressivement à distinguer ceux qui étaient portés par tous de ceux qui avaient vocation à signifier un statut particulier. Sur la tempe était incisé premièrement le motif commun à tous les Bwaba, dit de la «cithare». D'autres suivaient, au coin de la bouche, barrant de biais le plan de la joue. Réservées à quelques-uns, des lignes verticales ou hachurées reliaient la racine des cheveux à la mâchoire inférieure. De petites hachures sur le côté du front et d'autres marques, discrètes, désignaient des événements uniques, là la perte d'un jumeau décédé, ici l'exaucement d'un vœu émis par la parenté de l'enfant et regardant en général des affaires de fertilité. La jeune femme apprenait aussi que les visages des filles devaient, pour commencer, être marqués au menton du motif qui signifiait à la fois leur genre et l'une de leurs principales fonctions - préparer et cuisiner la nourriture -, le motif du «fouet à sauce ${ }^{4}$ ».

L'enseignement de la scarificatrice portait également sur les soins confidentiels indispensables à une cicatrisation harmonieuse des plaies, comprenant le lavage avec une décoction de galles bouillies recueillies sur une plante arbustive semée à cet effet sur le toit en terre recouvrant la forge (Guiera senegalensis) et connue pour ses propriétés antiseptiques dans le traitement des plaies, ainsi que l'introduction dans les incisions d'ingrédients qui n'avaient pas de pouvoir curatif particulier: des fragments de carapace de tortue, d'autres éléments de corps animaux, calcinés puis réduits en poudre, et de la suie prélevée sur les poteries de cuisine, tatouant définitivement les incisions d'un noir bleuté. Comme il est d'usage lorsque l'on soigne des plaies, celles-ci étaient ensuite enduites d'une couche de beurre de karité censée les protéger des infections.

Dans les années 1980, profitant d'une perte d'influence des forgerons dans la vie sociale, économique et politique, quelques femmes de cultivateurs décidèrent de procéder elles-mêmes au marquage du visage des enfants. Ne pouvant obtenir les lames traditionnelles forgées à cet effet, elles opérèrent avec des rasoirs Gillette qui provoquèrent divers accidents de cicatrisation. Maîtrisant mal et l'instrument et le geste, elles incisèrent maladroitement les motifs, faisant parfois des ratures en revenant sur un trait pour en modifier le dessin, défigurant presque leurs jeunes patients. Ce fut cependant moins le caractère malhabile des incisions 
qui leur attira la réprobation générale que le fait de s'être octroyées un droit qui ne leur revenait pas.

\section{L'art de composer}

La compréhension des règles de composition présidant au marquage des torses, lorsqu'il était profus, exigeait de la jeune apprentie qu'elle recoure à d'autres moyens mnémotechniques que ceux issus de la seule observation. La jeune fille ou le jeune homme se tenait en position agenouillée sur les talons face à la scarificatrice qui procédait, selon la demande, à l'incision de la poitrine, et éventuellement du ventre, ou du dos, les deux faces du torse n'étant jamais traitées dans la même journée. Lorsque toute la surface de la poitrine, du ventre ou du dos devait être gravée, le travail d'incision commençait toujours par le bas pour progresser vers le haut, afin que le sang coulant des blessures ne gêne pas sa progression ${ }^{5}$. II durait plusieurs heures quand l'image à réaliser était particulièrement compliquée et nécessitait un très grand nombre d'incisions.

Dans le cas particulier des images abondant sur le torse, je me posai longtemps la question de comprendre quelle était la représentation mentale qu'en avait la scarificatrice avant de se lancer dans l'inscription de ces multiples petits traits verticaux qui, une fois disposés en rubans, donnaient corps à des motifs singuliers, reconnus et nommés comme tels, recouvrant l'épiderme. Cette représentation devait être suffisamment précise et structurée pour lui permettre, malgré les écoulements de sang susceptibles de brouiller tout repère visuel, de donner forme à ces compositions en les incisant sans avoir à hésiter sur l'agencement des traits ${ }^{6}$. Pour m'être moi-même confrontée à l'épreuve de leur reproduction sur le support inerte et plan de la feuille de papier, j'ai pu approcher la difficulté de l'exercice, tout en ayant sous les yeux le modèle à recopier auquel je pouvais revenir en permanence pour guider ma main. Un dessinateur ou un peintre, face à la feuille ou la toile blanches, qu'ils soient ou non engagés dans la production d'une image d'après nature, élaborent mentalement leur composition par rapport à la configuration et aux dimensions du support sur lequel ils s'apprêtent à œuvrer, anticipant ainsi l'impact de leur geste. Un remords y est toujours permis: un trait de crayon s'efface, un coup de pinceau aussi. Rien de tel en revanche sur la peau, où toute hésitation est susceptible de laisser des traces disgracieuses et indélébiles. Rompue à l'analyse formelle des images, je m'aidai de ce savoir pour repérer dans ces motifs des axes et des divisions, dont je compris bientôt qu'ils en étaient la charpente, ce qui me permit d'effectuer mes relevés avec beaucoup plus d'aisance. Je tentai d'exposer aux scarificatrices ces principes organisateurs tels que je les avais perçus. Je ne fus pas comprise. Après bien des hésitations, elles me révélèrent unanimement par l'assertion suivante, «c'est la tortue qui a donné les wiini (scarifications) », que le modèle auquel elles se référaient pour agencer et disposer les motifs, et dont elles ne parlaient jamais, était celui des écailles de la dossière et surtout du plastron de la tortue [planche II]. La médiation de ce modèle matriciel de compréhension des images nous permit dès lors d'évoquer leur composition en nous appuyant sur une grille de lecture commune.
5. Dans les années 1970 chez les Mossi, voisins des Bwaba, Suzanne Lallemand a eu la possibilité d'assister à la scarification du ventre de deux jeunes filles. Les ethnologues africanistes ont peu écrit sur ce sujet, ce qui rend son témoignage d'autant plus précieux. En outre, l'article est accompagné de photographies prises lors de ces séances, documents rares s'il en est (Lallemand 1986) (fig. 4).

6. Les photographies prises par Suzanne Lallemand montrent que la scarificatrice mossi, avant d'inciser, dessinait à la craie le tracé de sa composition. L'auteur précise qu'il s'agissait d'un procédé récent (Lallemand 1986). Les femmes bwaba ne l'ont jamais évoqué en ma présence. 

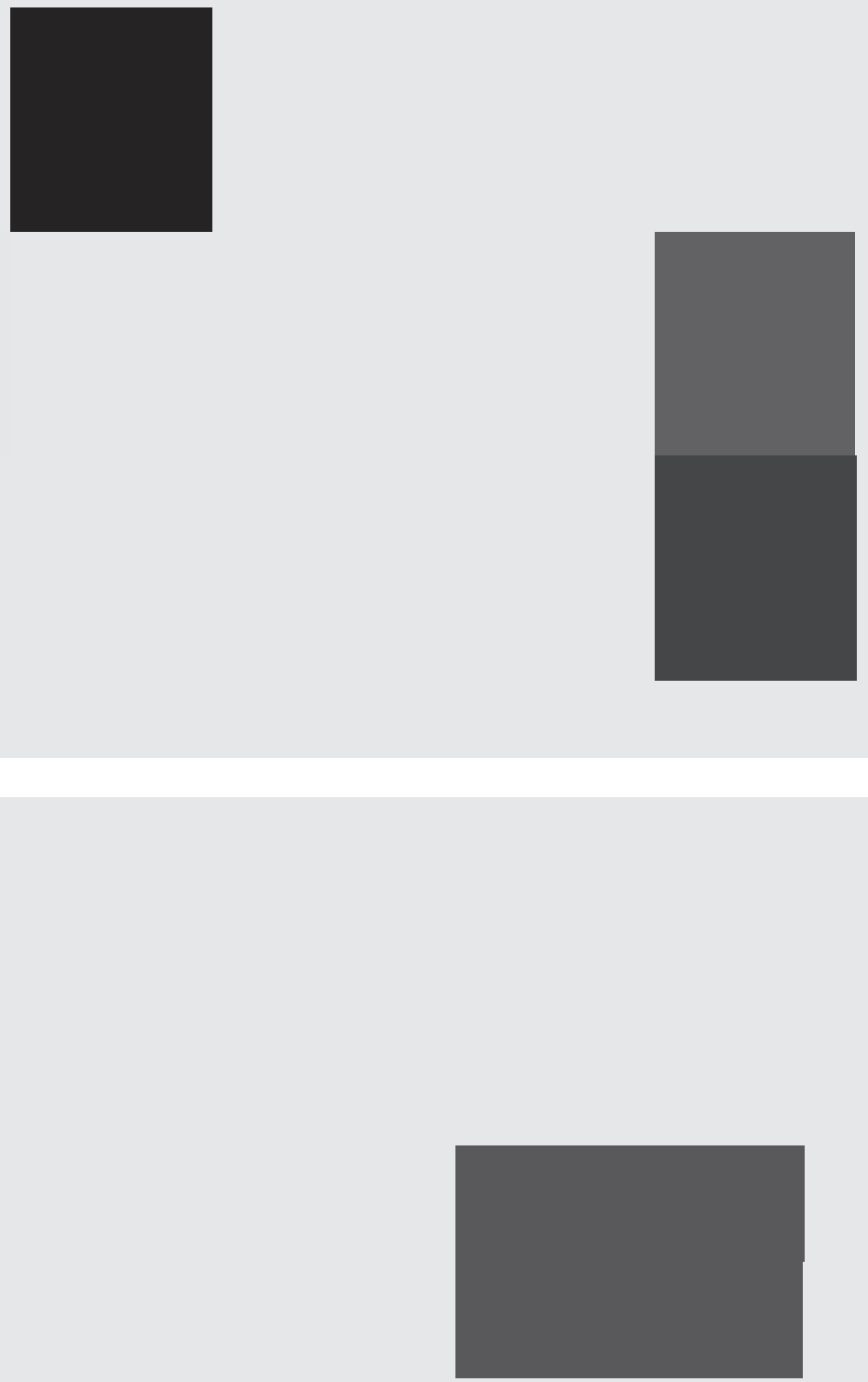
Le plastron de tortue constituait en réalité bien plus qu'un simple modèle graphique. II était l'archétype de toutes les images incisées sur les torses, quelles que soient leur densité et leurs dimensions. Le schéma simple d'accolement des écailles était facilement mémorisé par les scarificatrices, qui disposaient là d'un invariant fédérant structurellement l'ensemble de leurs réalisations. II importe de préciser ici que cet animal occupe une place essentielle ${ }^{7}$ dans la cosmologie élaborée par les forgerons, l'association qu'ils établissent entre tortue et scarifications n'étant pas uniquement la traduction d'une convention métaphorique. Les données relatives à la tortue dans les représentations autochtones et les pratiques rituelles concernant l'agriculture et la procréation révèlent que les motifs formés par les plaques cornées de la carapace sont décrits comme les modèles à suivre pour tracer tant les billons des champs de culture que les scarifications corporelles. Les femmes des forgerons se considèrent ainsi comme des travailleuses de l'épiderme au même titre que les cultivateurs sont des travailleurs de la terre. Graver la peau serait un geste semblable à celui de labourer le sol pour élever les billons en ce que l'un et l'autre œuvreraient à la fertilisation de la matière, qu'elle soit chair ou terre.

Des dessins figurant des personnages scarifiés, réalisés par un forgeron entraîné au maniement du stylo, montrent cependant que connaître cet archétype ne garantit pas qu'on soit capable de le traduire graphiquement sur un support; la transcription du tracé ne suit pas en effet le schéma de composition visible sur le plastron et reproduit l'agencement des scarifications des corps avec une certaine fantaisie (planche III).

La connaissance de ce modèle figurait parmi les multiples éléments constitutifs de la pratique de la scarification qui ne pouvaient être divulgués - au même titre que les actes et savoirs spécifiques comme la préparation des décoctions ou l'énonciation des paroles rituelles adressées aux puissances de la forge ainsi qu'aux ancêtres du lignage de cultivateurs dont étaient issus les enfants et les jeunes gens.

Ce canon est au fondement cognitif de la pratique des scarificatrices: sans lui, il leur eût été impossible de produire des images à la fois semblables dans leur composition et distinctes dans le remplissage de leurs différents champs; deux principes intangibles, le «même » signifiant l'appartenance à la communauté bwanou, le «différent», la singularité irréductible de chaque individu. Le recours caché à ce modèle, bien qu'étayé par un complexe édifice cosmologique, peut également être décrit comme le procédé mnémonique ingénieux, le truc en quelque sorte, essentiel à la production de ces images. Ces femmes en étaient parfaitement conscientes.

Par ailleurs, si elles connaissaient les noms des motifs et savaient où les placer sur le corps, elles ne pouvaient en aucun cas les commenter. Cela ne relevait pas de leurs compétences, l'exégèse revenant au seul doyen de la forge. J'ai pu vérifier à plusieurs reprises qu'elles en savaient évidemment beaucoup plus qu'elles ne pouvaient et ne voulaient en dire.

Lors de l'opération, en accord avec le doyen du lignage des cultivateurs, le maître de la forge précisait à la scarificatrice quels étaient les motifs
7. Ceci explique, en partie, la présence de fragments de carapace calcinés dans la poudre mélangée à la suie dont les blessures sont enduites.

\section{ci-contre \\ planche I \\ Relevé des scarifications du torse (face et dos) d'une femme de Mamboué. Dessin par Michèle Coquet.}

planche II

Dossière et plastron de tortue. Dessin par Michèle Coquet. 
8. Dont les dimensions n'excédaient pas $30 \mathrm{~mm}$ de longueur sur $15 \mathrm{~mm}$ de largeur environ, soit un instrument dont la préhension et la manipulation exigeaient une grande dextérité. à inciser en fonction de l'identité lignagère de l'enfant, de sa position dans la fratrie et d'autres éléments biographiques qu'il connaissait. Pour le marquage du torse des jeunes gens, le corpus graphique élaboré par les forgerons était en réalité peu étendu. Le doyen du lignage des forgerons mentionnait trois à quatre motifs à inscrire dans la peau, figurant à la fois des lieux et des acteurs nommés dans le récit de fondation du lignage et les lieux de culte relatifs à ladite fondation inscrits dans le territoire villageois (Coquet 2006).

Trois motifs sont à la base de toutes les compositions des images du torse, même les plus complexes: un ruban d'incisions tracé à l'horizontale ou à la verticale, un autre en ligne brisée et un dernier de chevrons. Ceux-ci suffisent à exprimer l'essentiel de ces histoires de fondation et l'identité lignagère de la personne qui les porte. L'association des rubans horizontaux et en ligne brisée, figurant des chemins et des collines, compose la représentation visuelle la plus simplifiée possible d'un récit de fondation. On retrouve cette formule graphique canonique, chemins et collines, dans toutes les images de torse. La répétition et l'invention d'agencements toujours renouvelés de ces deux motifs permettaient à la scarificatrice d'en produire d'autres: losanges, sabliers, triangles, quadrilatères... Le remplissage des champs s'obtenait par la multiplication des rangées de rubans ou l'adjonction de petits motifs réalisés à partir d'un dessin reposant toujours sur les mêmes principes graphiques de combinaisons de traits, faisant surgir un décor asymétrique et voulu comme tel.

\section{L'épreuve partagée du courage}

Par sa capacité à imaginer des compositions au décor prolifique et variant à l'infini, la scarificatrice révélait également ses talents créateurs. Dans ce cas cependant, il ne s'agissait pas tant pour elle de se faire plaisir, en compliquant sa composition plus que nécessaire, que de répondre au désir de sa patiente - rappelons que l'incision d'images profuses sur le torse ne concernait que les jeunes filles - d'endurer une scarification étendue de son épiderme. Quelques-unes de ces scarificatrices confiaient cependant qu'elles avaient pris plaisir à réaliser ces images dont l'exécution révélait, à elles-mêmes et aux autres, la qualité de la maîtrise de leur geste. Je pense aujourd'hui que cette confidence avait été induite par la question de l'ethnologue. Même dans ces cas particuliers, l'affirmation d'un plaisir de faire, provoquant paradoxalement de la douleur chez autrui, demeurait accessoire. En réalité, ce qui importait aux yeux des villageois, c'était la force morale que manifestaient les patientes comme les scarificatrices, célébrée en particulier lors de leurs funérailles.

Les chants composés pour l'occasion faisaient l'éloge du «travail» de la défunte, louant l'étendue du devoir accompli ainsi que son courage. "Elle a bien travaillé", disait-on des plus illustres scarificatrices longtemps après leur mort. L'épiderme orné des villageois en apportait chaque jour la preuve. Leurs outils, les petites lames avec lesquelles elles opéraient ${ }^{8}$, étaient exposés sur leur cadavre et destinés, selon la coutume, à être enterrés avec elles. Les lames étaient à ces femmes ce que l'arc, les flèches ou le fusil étaient aux grands chasseurs, le fouet à sauce aux femmes ayant donné naissance à une nombreuse descendance, la houe aux cultivateurs réputés pour leur ardeur au travail de la terre: plus que des insignes, 
ces objets relèvent pour les Bwaba d'une catégorie particulière en ce qu'ils sont voués à leur propriétaire puisque fabriqués expressément pour lui avant de lui être formellement remis. Aucun homme, aucune femme, ne donnerait, n'échangerait et encore moins ne vendrait son arc, son fouet à sauce ou sa houe. Ces objets sont considérés comme les bonna de leur possesseur, boni signifiant ordinairement l'«ombre» ou le «double» d'une personne. Cette qualification révèle ici l'intensité de la relation liant l'objet à celui qui le détient, construite par l'usage et éventuellement entérinée par le rite, équivalente à celle qui unit pour la vie tout être à son ombre - cette dernière adhérant à sa silhouette, reproduisant ses gestes, lui collant aux talons ${ }^{9}$. L'énoncé «elle a bien travaillé » signifiait que la scarificatrice avait accompli le devoir qui lui incombait, avait fait honneur à ses outils (les lames) et n'avait jamais failli à ses obligations.

Ne pas se dérober et affronter la peur comptait parmi les prescriptions auxquelles la scarificatrice et ses patients devaient se soumettre, alliés par une même adhésion à l'éthique collective du courage, alors pierre angulaire de la société bwanou. Pour les jeunes gens, en particulier pour les filles qui décidaient librement de rendre ce décor foisonnant en se faisant scarifier l'ensemble du torse et même les bras, faire montre de cette vertu à l'heure où la lame leur incisait la peau jusqu'en ses parties les plus tendres et les plus sensibles était une manière d'acquérir, aux yeux de toute la communauté, prestige et considération. Ces jeunes femmes étaient assurées d'exercer, au terme de l'opération, leur ascendant sur leurs pairs et sur les hommes: respect, admiration, pouvoir de séduction, beauté enfin leur étaient accordés ${ }^{10}$.

Les commentaires des Bwaba ne s'étendaient pas sur l'intensité de la douleur subie - «ça brûle comme le feu», disait-on sobrement ou sur les dangers d'infection. La réalité de ces derniers était même minimisée; seule la bravoure manifestée par le et surtout la patiente était évoquée dans les éloges, qu'ils aient été proférés dans l'intimité ou, sur un mode plus solennel, en public ${ }^{11}$. On attendait de la jeune fille mossi la même attitude vis-à-vis de la souffrance physique. Elle ne devait laisser transparaître aucun signe de faiblesse au moment de l'opération, ne proférer aucun cri ni reculer face au couteau. Sa «réputation est en jeu», écrit Suzanne Lallemand. «Peu endurante, une fille attriste sa mère, suscite le mépris des sœurs aînées et les moqueries des belles-sœurs; quelquefois la rumeur de sa médiocre performance peut l'accompagner jusque chez les femmes de la demeure d'un mari. » (Lallemand 1986: 69)

L'acte de la scarification, lorsqu'il portait sur l'ensemble du torse, provoquait des saignements de tout l'épiderme travaillé. Si cette perte de sang ne devait pas affecter les jeunes gens, elle ne devait pas non plus troubler la scarificatrice dans son ouvrage, tant d'un point de vue psychologique (faire couler le sang et son corollaire, faire souffrir) que pratique (ne pas dévier le trait): on disait d'elle qu'elle devait avoir le «cœur dur» pour résister à la vue du sang et à la souffrance qu'elle infligeait à autrui. Selon certaines praticiennes, l'excès de sang s'écoulant des blessures provoquait chez elles des désordres physiologiques importants: elles ne pouvaient plus aller à la selle, leur ventre «enflait» - un signe toujours considéré comme le symptôme d'une maladie grave - et leur absence d'appétit
9. Sur la catégorie du double dans cette aire culturelle, je renvoie à Coquet et Houseman 2006.

10. De fait, aux hommes il était bien peu demandé. Seul le visage pour la majorité d'entre eux était marqué, et ce, dans l'enfance. Pour douloureuse qu'ait été l'opération, elle n'exigeait pas la même vaillance.

11. Lors de la scarification de leur visage, les enfants pleuraient. Cris et plaintes n'étaient pas prohibés: comparés aux pleurs émis par les bébés à leur naissance, ils étaient conçus comme la manifestation d'une seconde «naissance», non plus biologique mais sociale. 


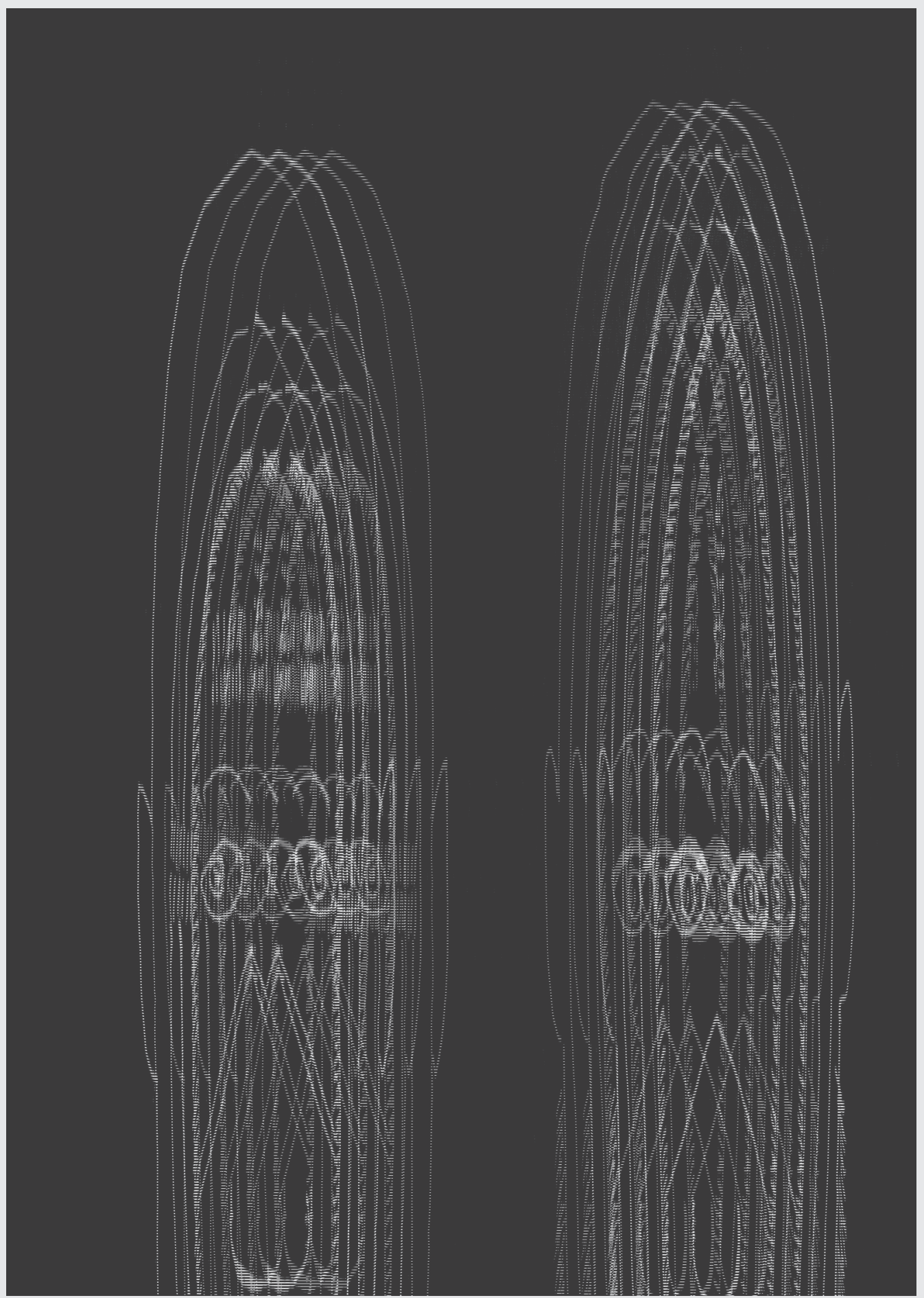


les contraignait à ne se nourrir que de bière de mil. Témoignant encore de l'étroite interaction entre la femme et ses patients, engagés à proportion égale dans le processus de marquage, un identique régime alimentaire leur était imposé, où toute nourriture suggérant par son aspect les conséquences d'une mauvaise cicatrisation était prohibée: la sauce de graines d'arachide parce que les fruits gonflés aux contours tordus ne manquaient pas d'évoquer des chéloïdes et la sauce de feuilles de baobab pour sa consistance gluante. Cette dernière rappelait l'aspect des plaies purulentes et éveillait la crainte d'obtenir pour tout résultat des motifs «mélangés» où les traits, après cicatrisation, n'auraient pas été bien distincts les uns des autres. La viande était également proscrite car elle contenait du sang animal dont le toucher et l'ingestion risquaient, par contact, de polluer les blessures des opérés.

\section{Les conditions d'émergence de la beauté}

L'épreuve de la douleur subie lors du marquage des torses transformait les jeunes filles en personnes d'exception suscitant l'admiration collective car elles avaient su puiser leur force intérieure pour supporter l'incision de leur épiderme. L'édification progressive du renom de la scarificatrice reposait sur les mêmes principes, la reconnaissance de son courage impliquant celle de la singularité de ses images, de leur style et de l'excellence de son savoir-faire. Ce dispositif relationnel où la douleur infligée et sa réponse, le courage, sont consacrées par la collectivité n'est pas propre à la scène des scarifications. Elle est au cœur du système initiatique: «ll ne s'agit pas seulement d'endurcir les novices en leur faisant subir des épreuves douloureuses, mais de prendre à témoin la société tout entière de leur souffrance. L'initié n'est pas seulement celui qui a souffert, mais celui dont tous savent - et dont quelques-uns ont vu - qu'il a souffert. II n'est devenu un être nouveau que parce que les autres en jugent ainsi; et eux fondent leur jugement sur les souffrances qu'ils l'ont vu endurer. » (Casajus 2005: 6) Dans le cas particulier qui nous intéresse ici, le surgissement d'un «être nouveau », par le truchement d'une opération mobilisant douleur, courage et volonté, ainsi que la possible (ce n'est pas toujours le cas) et progressive conversion d'un savoir-faire en geste porteur d'une singularité créatrice, sont deux processus constitutifs de l'apparition du sentiment esthétique. Car les images scarifiées, lorsqu'elles sont denses et bien faites, sont appréciées pour leur beauté.

En effet, les Bwaba, surtout les hommes, s'accordaient sur deux points: la beauté du corps des femmes aux torses gravés et le plaisir que les hommes avaient à caresser ces épidermes aux reliefs ténus et multiples. Ce consensus autour de la beauté était une manière d'hommage rendu à leurs épouses ou à leurs amantes, spécialement à celles dont le courage avait été suffisamment exceptionnel pour accepter une incision de tout le torse et des bras. Femmes et hommes en retiraient une fierté légitime. Cette présence sur le corps de chacun confirmait, au-delà des différences de statut ou de genre, l'appartenance à une culture commune, portée par des idéaux et des émotions esthétiques partagés: en ces images avait pris racine un même sentiment de la beauté. Les hommes ne manquaient pas non plus de commenter non seulement la grâce de leurs compagnes, mais aussi la science de celles qui l'avaient rehaussée par leur art. ci-contre

planche III Dessins du forgeron Tianhoun Didiiro, village de Kari, Burkina Faso. 
12. "To be in a satisfactory ritual state». Il est possible qu'il en ait été de même chez les Bwaba dans les années 1950. Trente ans plus tard, Iorsque je commençais à y séjourner, l'usage de cosmétiques comme l'huile de karité y était devenu rare et les villageois étaient tous vêtus.
Que l'ampleur de la souffrance subie et du courage requis pour y faire face magnifie la beauté du corps ainsi transformé était une idée également partagée par les Tiv du Nigeria. Paul Bohannan a insisté dans ses écrits sur les rapports entre la dimension esthétique des scarifications tiv et la valeur accordée au courage. II y rappelle que la beauté corporelle répond à cette conception selon laquelle chaque personne - et c'est une obligation sociale dont seuls les plus âgés sont exempts - doit se rendre séduisante et agréable à l'œil afin d'être regardée. Pour ce faire, les hommes et les femmes s'huilent ou se colorent le corps, se parent et se coiffent, se font tailler les dents d'une manière éventuellement inhabituelle et se font scarifier (Bohannan 1956: 117). Pour les Tiv, l'ornementation par scarification vient rehausser, ou parachever, le corps en ce qu'il doit luire ou rayonner, ce qu'ils traduisent par un terme, wanger, signifiant à la fois être beau en produisant de la "clarté" (to be clear) et «être dans un état rituel satisfaisant ${ }^{\mathbf{1 2}}$ » (ibid.).

Les Tiv précisent que la taille des dents est encore plus pénible à supporter que les scarifications. Et cette opération a plus de prix à leurs yeux car elle requiert davantage de courage alors qu'elle n'est pas exigée par la tradition; ainsi, mis au défi de subir cette opération par son amie qui le compare à un "crabe» ou un «larbin", un jeune homme peut décider de se faire tailler les dents. II prend alors soin d'être accompagné par des camarades chargés ensuite de témoigner auprès de la jeune fille de son impassibilité face à la douleur (ibid.: 120). La jeune femme qui exhibe dans un large sourire ses incisives comprenant chacune une encoche, de sorte que chaque dent semble dédoublée, se sait admirable et admirée à la fois pour le spectacle de sa denture démultipliée et pour la bravoure dont elle a fait preuve en en subissant la sculpture. La beauté de son sourire n'en devient que plus fascinante. L'effet est tel, rapporte l'auteur, que chacun éprouve le besoin de la regarder à plusieurs reprises: les Tiv en concluent que la jeune fille est si rayonnante, si pleine de wanger, qu'elle ne peut que devenir le centre de l'attention.

Wiini est le terme par lequel les Bwaba nomment les scarifications. Le pluriel désigne les traces dues au passage d'un objet pointu sur une surface plane causées en principe par une incision ou un procédé assimilé, comme lorsque la potière utilise un épi de maïs pour imprimer un décor dans la terre molle. Ce terme n'est cependant pas utilisé pour qualifier les empreintes laissées par les êtres vivants sur le sol (laa). Les dessins effectués avec un burin de fer sur du métal, avec une herminette sur le bois, avec une lame de fer dans la peau se nomment donc wiini. Mais wiini se rapporte aussi à d'autres graphismes: ceux creusés par l'insecte dans le bois, ceux que dessinent les nervures de la feuille, les écailles du python ou les plaques cornées de la carapace de la tortue, la ligne aveuglante de l'éclair dans le ciel, les lignes et les traits surgissant au passage du stylo sur une feuille et, dans certains contextes, les sillons des champs. Le même terme peut servir à décrire une figure linéaire réalisée en volume: on dira des vertèbres, alignées pour composer la colonne vertébrale, qu'elles sont des wiini. Lorsque les Bwaba désignent par ce mot les nervures de la feuille ou les vertèbres, c'est pour ce qu'il permet de nommer sur le plan formel (des réseaux de lignes ou de traits répétés) ou fonctionnel 
(une armature qui maintient ensemble un assemblage de parties distinctes). Ce terme désigne donc à la fois des éléments identiques disposés linéairement - vertèbres, petits traits des scarifications, nervures des feuilles, caractères d'écriture ou d'imprimerie... - et les images composées de traits assemblés dont chacun opère comme une césure sur une surface, tel le motif scarifié dans l'étendue de la peau ou l'éclair dans la vastitude du ciel. Les wiini apparaissent ainsi comme des ensembles organisés de lignes et de traits se détachant sur une surface constituant, à la manière d'une charpente, le bâti de certains objets du monde.

La régularité des incisions, de même longueur, leur parallélisme, l'égalité des espacements entre chacune, la densification contrôlée du décor et l'équilibrage de leurs divisions, toutes qualités plastiques témoignant de l'habileté de la scarificatrice, portent à son expression magnifiée parce que parfaitement maîtrisée ce que, au-delà des images scarifiées, incluent l'emploi du terme wiini et sa matérialisation dans la chair: une théorie autochtone de la phylogenèse des choses du monde, des êtres vivants, des phénomènes naturels et des matières, conçus non pas du point de vue de leur apparence mais de ce qui les structure et les construit. Les wiini exprimeraient l'achèvement de ce processus. C'est aussi ce à quoi s'emploie la scarificatrice: porter la touche finale aux corps de ses semblables en scarifiant leurs visages et leurs torses. Pour certains, ou plutôt certaines, ce geste conduit à la naissance d'un être non seulement nouveau mais plus accompli que les autres. 


\section{Boas, Franz}

2003 (1927) L'Art primitif.

Paris, Adam Biro.

\section{Bohannan, Paul}

1956 «Beauty and scarification amongst the Tiv », Man 56: 117-121.

\section{Casajus, Dominique}

2005 «Présentation",

in L'Excellence de la souffrance, Systèmes de pensée en Afrique noire $17: 5-24$

\section{Coquet, Michèle}

1994 "L'envers du regard", Journal des africanistes 64(2): 39-63.

2006 "L'image et sa glose: composition graphique, structure narrative et énonciation ",

in Simon Battestini (dir.),

De l'écrit africain à l'oral.

Le phénomène graphique africain. Paris, L'Harmattan : 89-109.

\section{Coquet, Michèle et Houseman, Michael}

2006 "Le corps et ses doubles", in Stéphane Breton (dir.)

Qu'est-ce qu'un corps? Afrique de l'Ouest, Europe occidentale, Nouvelle-Guinée, Amazonie. Paris, musée du quai Branly-Flammarion: 25-57.

\section{Lallemand, Suzanne}

1986 «Entre excision

et accouchement: les scarifications des filles mossi du Burkina", Archiv für Völkerkunde 40: 63-74. 


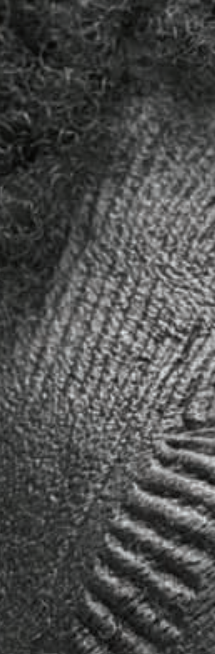

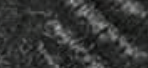

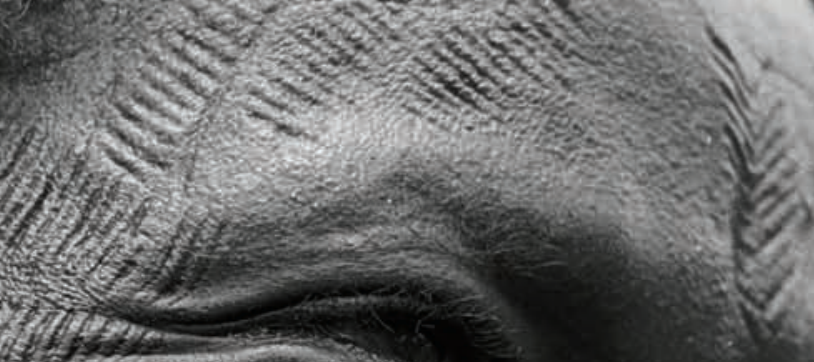

40

4.

7 .

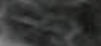

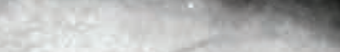

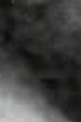

